\title{
Die Außenpolitik der Vereinigten Staaten von Amerika und ihr Bekenntnis zum multilateralen Internationalismus
}

\section{Thomas Salzmann}

Kerngebiet: Zeitgeschichte

eingereicht bei: Ass. Prof. in Mag. ${ }^{a}$ Dr. ${ }^{\text {in }}$ Eva Pfanzelter, MA

eingereicht im Semester: SS 2013

Rubrik: BA-Arbeit

\begin{abstract}
U.S. Foreign Policy and its Commitment to Multilateral Internationalism

In this paper it will be argued that the Truman and the Eisenhower Administration, in dealing with post-war Germany, the Soviet Union and the Cold War, followed a policy of multilateral internationalism. After introducing this concept in the first part, the second part will deal with the question whether the reorientation that George W. Bush brought about in U.S. foreign policy has fundamentally altered its basic focus on multilateral internationalism. Based on an analysis of Barack Obama's foreign policy, it will be argued against such an alleged profound change.
\end{abstract}

\section{Einleitung}

Der multilaterale Internationalismus ist eine Form der US-Außenpolitik, die aus der Frage erwuchs, wie nach dem Zweiten Weltkrieg mit der Sowjetunion und vor allem mit dem besiegten Deutschland umzugehen sei. Eine Analyse der deutschen Verhältnisse in den Jahren nach dem Ersten Weltkrieg offenbarte eine Reihe von Fehlern der Siegermächte, die es nach 1945 zu vermeiden galt. Dem amerikanischen Präsidenten Woodrow Wilson war es nach dem Kriegsende 1918 nicht gelungen, die von Frankreich und Großbritannien betriebene Isolierung der nach dem Ende des Deutschen Kaiserreichs begründeten Weimarer Republik zu verhindern. Die dann der Weimarer Republik im Frieden von Versailles auferlegten Verpflichtungen, vorneweg die umfassenden Reparationszahlungen und die Anerkennung der Kriegsschuldklausel, wurden zum Nährboden für radikale politische 
Strömungen, boten sie doch Parteien wie der NSDAP oder der KPD die Möglichkeit, diese für die eigenen politischen Heilsvorstellungen zu instrumentalisieren. Adolf Hitler, am 30. Januar 1933 zum Reichskanzler ernannt, verwandelte das noch junge demokratische Deutschland in einen totalitären Staat, an dessen Spitze stehend er am 1. September 1939 schließlich den Zweiten Weltkrieg entfesselte.

Dieser Rückblick führte bei den USA zu zwei Erkenntnissen, wie mit dem 1945 wirtschaftlich und politisch am Boden liegenden Deutschland umzugehen sei. Es war einerseits offensichtlich, dass es 1933 ein physisch wie auch psychisch geschwächtes Land war, das Hitler an die Macht verholfen hatte. Nicht minder augenscheinlich waren das politische und militärische Potenzial, wie auch der nationalistische Charakter dieses Staates am Ausgang des Zweiten Weltkriegs kaum zu übersehen war. Die USA zogen daraus zwei Schlussfolgerungen, dass nämlich erstens ein ökonomisch stark geschwächtes Deutschland für radikale Tendenzen anfällig war und dass zweitens ein nationalistisch ausgerichtetes, politisch und militärisch mächtiges Deutschland eine Gefahr für Europa darstellte. Darüber hinaus offenbarte die Analyse der europäischen gesamtwirtschaftlichen Situation zu Zeiten der Weimarer Republik, dass Europas Gesundung ohne eine Genesung Deutschlands nicht möglich war.

Die Herausbildung dieser Einsichten wurde in der Nachkriegszeit von zunehmenden Meinungsverschiedenheiten zwischen den westlichen Alliierten und der Sowjetunion begleitet, die den fragilen Charakter der Anti-Hitler-Koalition offenlegten, sie als reines Zweckbündnis entlarvten und die USA zwang, sowohl für den Umgang mit Deutschland, als auch der Sowjetunion Lösungen zu finden. Die beiden Problemsphären konnten und können unmöglich als voneinander getrennt analysiert werden, allein schon deshalb, weil die Sowjetunion eine der Besatzungsmächte Deutschlands war. Die konkrete, aus amerikanischer Sicht als Wiederholung der Ereignisse von 1933 unter verkehrten Vorzeichen zu sehende Gefahr bestand darin, "dass Hunger, Armut und Verzweiflung die Europäer veranlassen könnten, ihre eigenen Kommunisten in die Regierung zu wählen, die dann gehorsam den Moskauer Wünschen folgen würden"1. Um ein solches Szenario zu vermeiden, beschlossen die USA auf eine ökonomische Stabilisierung Deutschlands und Europas hinzuarbeiten.

Die schließlich ergriffenen wirtschaftspolitischen Maßnahmen waren Teil eines außenpolitischen Gesamtkonzeptes der USA, das als Eindämmung (Containment) der Sowjetunion in die Geschichtsbücher Eingang fand. In diesen Überlegungen nahm Westeuropa aufgrund seiner geographischen Nähe zum heraufziehenden Eisernen Vorhang („,iron curtain"2) eine besondere militärstrategische Funktion ein, die zu erfüllen aber erst dann vorstellbar war, wenn die einzelnen westeuropäischen Länder ökonomisch wie auch politisch stabilisiert worden waren. Die politische Stabilisierung Deutschlands galt es derart zu gestalten, dass das Land nicht erneut zur Gefahr für Europa und die Welt wurde. Um diesen Spagat durchzuhalten, Deutschland einerseits zu kontrollieren und

1 John Lewis Gaddis, Der Kalte Krieg. Eine Neue Geschichte, München 2007, S. 47.

2 Steven James Lambakis, Winston Churchill. Architect of Peace. A Study of Statesmanship and the Cold War (Contributions in Political Science), Westport 1993, S. 85. 
andererseits als Bollwerk gegen sowjetische Ambitionen in Europa zu etablieren, mussten schon im Zuge der ökonomischen Konsolidierung politische Strukturen geschaffen werden, die auf mittel- und langfristige Sicht eine wirtschafts- und sicherheitspolitische Einbindung bzw. Integration des besetzten Landes erlaubten. Die letzten Endes geschaffenen wirtschafts- und militärpolitischen Strukturen waren der Marshall-Plan, der Schuman-Plan und die Europäische Gemeinschaft für Kohle und Stahl (EGKS), die Europäische Verteidigungsgemeinschaft (EVG) und die North Atlantic Treaty Organization (NATO). Das diese Institutionen einigende Band ist der Multilateralismus.

Die Beschreibung der amerikanischen Außenpolitik als multilateralen Internationalismus meint demnach die Betonung multilateraler Strukturen und Bündnissysteme auf internationaler Ebene zum Zwecke des Erreichens der eigenen Ziele. Die Verfechter des Pendants, des unilateralen Internationalismus, bestehen darauf, "that the United States ha[s] to preserve a free hand to act abroad"3. Die Analyse der Außenpolitik George W. Bushs, obwohl auch internationalistisch im Charakter, wird eine deutliche Entfremdung vom multilateralen Internationalismus Trumans und seiner Berater, der sogenannten "Wise Men"4 aufzeigen.

Die leitende Frage der vorliegenden Arbeit lautet, ob Bush Juniors Neuausrichtung der USAußenpolitik deren bisherige multilaterale Form grundlegend und auf Dauer veränderte.

Nach einer Analyse des Charakters von Barack Obamas außenpolitischem Agieren, wird die These bewertet, ob und wenn ja inwiefern die Außenpolitik seines Vorgängers lediglich eine temporäre Abweichung vom multilateralen Internationalismus war, wie er sich nach dem Zweiten Weltkrieg herauskristallisiert und auch während des Kalten Krieges weitgehend seine Gültigkeit beibehalten hatte.

Die Begriffe des Isolationismus und Internationalismus sind fester Bestandteil von Diskursen zur US-Außenpolitik. So hätten die USA unter Franklin D. Roosevelts Ägide etwa einen „internationalist turn"5 vollzogen. Der Ausdruck des Internationalismus ist aber problematisch, weil viel zu weit und unpräzise gefasst, und folglich „adds little of value to any consideration of US foreign policy"6. Genauer ist der Terminus des multilateralen Internationalismus, der in dieser Arbeit Verwendung findet. Dabei liegt der Fokus weniger auf dem Umgang der USA mit den internationalen Institutionen als solchen, sondern stärker auf den bilateralen Beziehungen mit den einzelnen europäischen Verbündeten, die ebenfalls Mitglieder der entsprechenden Organisationen waren. Da der multilaterale Internationalismus wesentlich von der amerikanischen Deutschlandpolitik nach dem Zweiten Weltkrieg geprägt wurde, bildet diese auch in den Kapiteln über George W. Bushs und Barack Obamas Außenpolitik ein wenig den roten Faden.

3 Ivo H. Daalder/James M. Lindsay, America unbound. The Bush revolution in foreign policy, New Jersey 2005, S. 7

4 Walter Isaacson/Evan Thomas, The Wise Men. Six Friends and the World they Made. Acheson, Bohlen, Harriman, Kennan, Lovett, McCloy, New York 1986.

5 Peter Trubowitz, Regional Shifts and US foreign policy, in: US Foreign Policy, hrsg. v. Michael Cox/Doug Stokes, New York 2008, S. 145-162, hier S. 152.

6 Andrew Johnstone, Isolationism and internationalism in american foreign policy, in: Journal of Transatlantic Studies 9 (2011), Nr. 1, S. 7-20, hier S. 14, [http://www.tandfonline.com/doi/pdf/10.1080/14 794012.2011.550772\#.VRLjK-Edldw], eingesehen 06.10.2013. 
Nach Stephen F. Szabo liegt die Ursache für die Kluft im deutsch-amerikanischen Verhältnis in einer Divergenz der "strategic cultures” der jeweiligen Länder. Der bezeichnende Titel von Szabos 2004 veröffentlichter Monographie lautet denn auch "Parting Ways" maßgeblichen Wandel der US-Außenpolitik hatten H. Daalder und James M. Lindsay in ihrer Analyse "America Unbound. The Bush Revolution in Foreign Policy"8 bereits im Vorjahr konstatiert, worin innen James Mann in "Rise of the Vulcans" folgte. Ohne dass sich diese Analysen des Terminus vom multilateralen Internationalismus bedienen, ermöglichen sie doch den Leserlnnen die Erkenntnis, dass sich Bushs Außenpolitik grundlegend von diesem abgewandt hatte.

Zwar existieren bereits ähnlich umfassende Analysen zur Außenpolitik Barack Obamas, doch stellt das Interagieren mit internationalen Organisationen und anderen Regierungen wenig mehr als eine Randnote dar. So erwähnt etwa David E. Sanger in seinem Buch "Confront and Conceal" 10 andere Staaten nur, wo diese in irgendeiner Form an militärischen Unternehmungen beteiligt waren. Nichtsdestotrotz entsteht bei der Lektüre von Sangers Werk ein wenig der Eindruck, als ob Obama Kooperation und Bündnisstrukturen wieder stärker in den Mittelpunkt der Außenpolitik gerückt hatte. Darauf deuten auch die Überschrift von H. Daalders und James G. Stravridis' in Foreign Affairs erschienenem Artikel, "NATO's Victory in Libya"11, und James Manns Ausführungen zum Militäreinsatz in Libyen in "The Obamians” hin.

Diese Ausarbeitung ist dergestalt untergliedert, dass vorneweg das Konzept der Eindämmung untersucht wird, wobei eine Analyse der konkreten politischen Strukturen (Marshall-Plan, etc.) der Eindämmungs- und Einbindungspolitik der Sowjetunion, respektive Deutschlands das Porträt der US-Außenpolitik während der Nachkriegszeit abrundet. Hierauf folgend werden die Außenpolitiken George W. Bushs und Barack Obamas untersucht, wie sie sich im Umgang mit so einschneidenden Ereignissen und Entwicklungen wie dem 11. September 2001, dem Krieg gegen den Terrorismus und im Irak und der "Arabische[n] Revolution"12 manifestierten. Abschließend wird eine Bewertung von Fragestellung und These vorgenommen.

Methodisch handelt es sich bei vorliegender Arbeit also um eine Analyse des Charakters der US-Außenpolitik während der Präsidentschaften Trumans, Eisenhowers, Bushs und Obamas, wobei die Herausbildung und Entwicklung des multilateralen Internationalismus anhand mehrerer Ereignisse nachvollzogen wird.

Von einem Quellenmangel zur Geschichte der US-Außenpolitik und der transatlantischen Beziehungen der vergangenen rund 70 Jahre kann keine Rede sein.

$7 \quad$ Stephen F. Szabo, Parting Ways. The crisis in German-American relations, Washington D. C. 2004, S. 52.

8 Ivo H. Daalder/James M. Lindsay, America Unbound. The Bush Revolution in Foreign Policy, New Jersey 2005.

9 James Mann, Rise of the Vulcans. The History of Bushs's War Cabinet, New York 2004.

10 David E. Sanger, Confront and Conceal. Obama's Secret Wars and Surprising Use of American Power, New York 2012.

11 Ivo H. Daalder/James G. Stavridis, NATO's Victory in Libya. The Right Way to Run an Intervention, o. D., [http://www.foreignaffairs.com/articles/137073/ivo-h-daalder-and-james-g-stavridis/natos-victory-in-li bya], eingesehen 06.10.2013.

12 Frank Nordhausen/Thomas Schmid (Hrsg.), Die Arabische Revolution, Berlin 2011. 
Daher stützt sich diese Arbeit überwiegend auf Sekundärliteratur namhafter Autoren, die als Instanzen auf ihren Gebieten gelten können, was etwa dadurch festgestellt wurde, dass auch andere Wissenschaftler in ihren Arbeiten auf diese Bezug nahmen bzw. auf sie verwiesen. Die wenigen herangezogenen Primärquellen dienen einmal dem Ziel einer möglichst eng am Material ausgerichteten Analyse und daneben der qualitativen Überprüfung von Sekundärquellen.

\section{Entstehung des Eindämmungskonzepts}

Rund einen Monat vor Japans Angriff auf Pearl Harbour, im November 1941, veröffentlichte der Geschichtsprofessor Dexter Perkins einen Artikel mit der Überschrift "Fundamental Principles of American Foreign Policy". Die ersten drei der von Perkins herausgearbeiteten Leitsätze diktierten demnach die amerikanische Europapolitik, wobei sich der erste darauf bezieht, dass die USA feste Allianzen (entangling alliances) mit europäischen Mächten vermieden. Das zweite Prinzip betont die vollständige Neutralität in europäischen Kriegen und das dritte Leitmotiv lässt sich mit dem Terminus "freedom of the seas" greifen. ${ }^{13}$ Der Kriegseintritt im Jahre 1917 verstieß zwar gegen den zweiten Leitsatz, doch muss die Ablehnung des Völkerbundes durch den US-Kongress als Rückkehr zum status quo ante gesehen werden. Der Terminus "freedom of the seas" beschreibt im engeren Sinne das Konzept des Freihandels, bringt in einem umfassenderen Sinne aber auch die Vorstellung einer freien Welt zum Ausdruck, was wiederum als Argument für einzelstaatliche Souveränität interpretiert werden kann. Der amerikanische Präsident Franklin D. Roosevelt hing diesen Vorstellungen an, mit denen er die Hoffnung verknüpfte, dass auch die Sowjetunion an einer freien Welt interessiert sei. Nach Roosevelts Tod im April 1945 verfolgte sein Amtsnachfolger Harry S. Truman noch für einige Zeit eine dementsprechende Politik, verkündete dann aber, durch das sowjetische Verhalten ernüchtert, am 12. März 1947 „die freien Völker zu unterstützen, die sich [...] Druck von außen widersetzen"14. Die Truman-Doktrin war geboren.

Hiermit ging ein grundsätzlicher Wandel der bis dahin weitgehend unbestrittenen außenpolitischen Prämissen der US-Außenpolitik einher, verschrieb sich diese doch nunmehr einer Integration in „entangling alliances” und opferte damit den Isolationismus dem multilateralen Internationalismus. Dieser strategische Richtungswechsel war notwendige Voraussetzung einer Politik von Eindämmung und Einbindung. Die Politik der Eindämmung ist als Reaktion auf sowjetische expansionistische Tendenzen zu verstehen, wie sie in der Besetzung osteuropäischer Staaten, aber auch der gescheiterten Einverleibung des Iran zum Ausdruck kamen. Die der Eindämmung zugrundeliegenden konzeptuellen Überlegungen waren im Februar 1946 von George F. Kennan in seinem „langen Telegram" entwickelt worden. Kennan kam nach seiner Analyse der

13 Dexter Perkins, Fundamental Principles of American Foreign Policy, in: Annals of the American Academy of Political and Social Sciences 218 (1941), Heft 6, S. 9-19, hier S. 9-15, [http://ann.sagepub.co m/ content/218/1/9.extract], eingesehen 26.04.2013.

14 Zit. n. Gaddis, Der Kalte Krieg, S. 46; Melvyn P. Leffler, The emergence of an American grand strategy, 1945-1952, in: The Cambridge History of the Cold War I, hrsg. Melvyn P. Leffler/Odd Arne Westad, Cambridge 2010, S. 67-88, hier S. 67-68. 
sowjetischen Regierungspraxis zu dem Schluss, dass diese als Ergebnis einer Symbiose von innerrussischen historischen Zusammenhängen mit der kommunistischen Ideologie, welche von der Unvereinbarkeit des kapitalistischen und kommunistischen Systems ausgehe, zu bewerten sei. ${ }^{15}$

Diese Erkenntnisse rundete Kennan in einem im Juli 1947 in Foreign Affairs (unter dem Pseudonym "X") publizierten Artikel mit dem Titel "The Sources of Soviet Conduct” ab, in dem er betonte, dass die USA gegenüber der Sowjetunion auf "Iong-term, patient but firm and vigilant containment of Russian expansive tendencies" setzen müssten. ${ }^{16}$ Dean G. Acheson, zu jener Zeit Unterstaatssekretär im State Department, kommentierte Kennans "Ianges Telegram" mit den Worten, "[that] his predictions and warnings could not have been better". Jedoch: "We responded to them too slowly"17. Damit bezog er sich wohl auf den Umstand, dass Kennans Überlegungen erst rund ein Jahr später, nämlich in Trumans Rede vom 12. März 1947 in die Form einer konkreten außenpolitischen Strategie gegossen wurden. ${ }^{18}$

Es war nicht nur die militärische Bedrohung durch die Sowjetunion, die Truman zum Handeln zwang, sondern auch die katastrophale gesamtwirtschaftliche Situation Europas und der deutschen Besatzungszonen zu Beginn des Jahres 1947. So waren zwar bis Ende 1946 Fortschritte erzielt worden, doch der harte Winter von 1946 auf 1947 führte dazu, dass Schnee und Eis die „vorher verzeichnete[n] Gewinne fast zunichte” machten. ${ }^{19}$ Das Transportwesen kam zum Erliegen, die Kohle wurde knapp, die Produktion brach ein und es kam zu einer Versorgungskrise. ${ }^{20}$

Dass diese ökonomisch angespannte Situation auch politisch gefährlich war, davon waren Angehörige der US-Armee überzeugt, die warnten, dass in dieser prekären Lage der Kommunismus weiter an Boden gewänne. Im Mai 1947 mahnte dann auch der Unterstaatssekretär für wirtschaftliche Angelegenheiten im State Department, William L. Clayton, dass „without further and substantial aid from the United States, economic, social, and political disintegration will overwhelm Europe"21.

15 George F. Kennan, 861.00/2 - 2246: Telegram. The Charge in the Soviet Union (Kennan) to the Secretary of State, o.D., [http://www2.gwu.edu/ nsarchiv/coldwar/documents/episode-1/kennan.htm], eingesehen 11.05 .2013$.

16 X, The Sources of Soviet Conduct, in: Foreign Affairs 25 (1947), Nr. 4, S. 566-582, hier S. 575, [http:// www.jstor.org/discover/10.2307/20030065?sid=21 106240177913\&uid=38722\&uid=591021 6\&uid= 67 \&uid=62\&uid=38721 \&uid=3\&uid=70\&uid=2\&uid=2134\&uid=3737864\&uid=5910616], eingesehen 12.05.2013.

17 Dean Acheson, Present at the creation. My years in the State Department, New York 1987, S. 151.

18 Charles S. Maier, Es geht um die Zukunft Deutschlands und damit um die Zukunft Europas, in: Deutschland und der Marshall-Plan, hrsg. v. Charles S. Maier/Günter Bischof, Baden-Baden 1992, S. 13-52, hier S. 18.

19 Michael J. Hogan, Europäische Integration und deutsche Reintegration: Die Marshallplaner und die Suche nach Wiederaufbau und Sicherheit in Europa, in: Deutschland und der Marshall-Plan, hrsg. v. Charles S. Maier/Günter Bischof, Baden-Baden 1992, S. 139-199, hier S. 142.

20 Maier, Es geht um die Zukunft Deutschlands, S. 18.

21 Leffler, The emergence of an American grand strategy, S. 74. 


\section{Vier Bündnissysteme zur Einbindung Deutschlands}

\section{Der Marshall-Plan}

Es war Aufgabe des neuen amerikanischen Außenministers George C. Marshall diese politischen und wirtschaftlichen Schwierigkeiten in den Griff zu bekommen. Zur Zeit seines Amtsantritts Anfang 1947 liefen zwar bereits auf bilateraler Ebene Hilfsprogramme, wie GARIOA ("Government Aid and Relief in Occupied Areas"), in dessen Rahmen bis dahin bereits 840 Millionen Dollar allein an Deutschland gezahlt worden waren, doch "die Sache blieb unbefriedigend und reichte nicht aus"22. Erschwert wurde Marshalls Aufgabe durch den Beschluss der Siegermächte auf der Potsdamer Konferenz im Sommer 1945, Deutschland zwar in vier Besatzungszonen zu teilen, das Land aber nichtsdestotrotz als wirtschaftliche Einheit zu behandeln. Die Widersprüchlichkeit dieser Regelung erkannten die amerikanischen Verantwortlichen erst eineinhalb Jahre später im Zuge der Moskauer Außenministerkonferenz, als sie bei dem Versuch, die wirtschaftliche Gesamtsituation Deutschlands in einem umfassenderen Rahmen anzugehen, auf vehementen Widerstand Frankreichs und der Sowjetunion stießen, die Deutschland harte Bedingungen auferlegen wollten. Spätestens zu diesem Zeitpunkt erkannten Marshall und seine führenden Mitarbeiter, Dean G. Acheson, George F. Kennan und William L. Clayton, „that German economic growth was vital to European economic recovery as a whole"23.

Marshalls Planungen hatten nichts mehr mit früheren Hilfsmaßnahmen gemein. Das Ziel war die Wiederbelebung des gesamten europäischen Wirtschaftsraumes und nicht mehr nur bilaterale Finanzspritzen, in der Hoffnung, die begünstigten Staaten würden dann ganz von allein wieder auf die Beine kommen. Bei dem am 5. Juni 1947 verkündeten European Recovery Program, später als Marshall-Plan bezeichnet, handelte es sich zunächst aber um wenig mehr als einen Vorschlag an die vor allem europäische Staatenwelt. Stellvertretend für diese berieten Ende Juni in Paris auf der sogenannten „Dreierkonferenz" Frankreich, Großbritannien und die Sowjetunion über Marshalls Angebot. Der sowjetische Außenminister Molotow verweigerte schließlich eine Partizipation, schien er doch zu befürchten, dass der gesamteuropäische Ansatz des Marshall-Planes und der damit einhergehende Einfluss der USA in die inneren Angelegenheiten der Sowjetunion die Souveränität seines Landes verletzen könnte. ${ }^{24}$

Ein allzu direktes Eingreifen war zu Beginn aber keineswegs Marshalls Absicht. Vielmehr wünschte er, "daß Teilnehmerländer die Initiative ergreifen und die Verantwortung für die Gestaltung eines Aufbauprogramms übernehmen", die Vereinigten Staaten hingegen Rahmenbedingungen schaffen und die Zahlungsvoraussetzungen definieren würden. Hierzu gehörte etwa, dass Zahlungsempfänger sich zum Multilateralismus und der Einbindung Deutschlands verpflichten mussten. Dementsprechend beschlossen noch in

22 Maier, Es geht um die Zukunft Deutschlands, S. 19.

23 William I. Hitchcock, The Marshall Plan and the creation of the West, in: The Cambridge History of the Cold War I, hrsg. Melvyn P. Leffler/Odd Arne Westad, Cambridge 2010, S. 154-174, hier S. 155.

24 Hogan, Europäische Integration, S. 150-151. 
Paris Großbritannien und Frankreich das Committee on European Economic Cooperation (CEEC), aus dem ein Jahr später die Organization for European Economic Cooperation (OEEC) hervorging. ${ }^{25}$

Frankreich und Großbritannien aber schienen vom Rezept des Multilateralismus nicht recht überzeugt zu sein. So forderte der britische Außenminister Ernest Bevin von Unterstaatssekretär William L. Clayton immer wieder, Großbritannien möge doch bilaterale Finanzhilfen zur Verfügung gestellt bekommen, denn schließlich „herrsche [es] über ein Weltreich, führe wichtigen Handel mit nichteuropäischen Ländern und trüge eine schwere finanzielle Last in Deutschland". Clayton überzeugte diese Argumentation nicht, er behandelte Bevins Heimatland wie „irgendein europäisches Land"26.

Neben den britischen Wunsch nach Sonderbehandlung trat die Furcht der Franzosen, dass eine Integration Deutschlands ihren Sicherheitsinteressen zuwiderlaufen könnte. Beide Länder bremsten infolgedessen die CEEC-Verhandlungen aus und erst auf stärkeren Druck der USA hin kam es zu deren Abschluss. In den Vereinigten Staaten selbst erhielt im April 1948 der Marshall-Plan mit der Verabschiedung des European Recovery Act den Segen des Kongresses. Der gleichzeitig ratifizierte Foreign Assistance Act legte die Grundlage zur Institutionalisierung des Marshall-Planes und bestimmte zugleich, dass ,jedes Teilnehmerland de[s] Marshall-Planes [...] einen bilateralen Vertrag mit den Vereinigten Staaten zu unterzeichnen" habe. ${ }^{27}$ Mit diesem Mittel waren die USA in der Lage sicherzustellen, dass die Empfängerländer die mit den Zahlungen verknüpften Bedingungen auch erfüllten. Parallel zu diesen Meinungsverschiedenheiten zwischen den USA und Großbritannien und Frankreich spielten sich Konflikte zwischen dem State Department und der amerikanischen Militärregierung in Deutschland unter General Lucius D. Clay ab, der die Befürchtung gehegt hatte, das besetzte Land würde beim Ansatz des gesamteuropäischen Wiederaufbaus auf der Strecke bleiben. ${ }^{28}$

Diese Konflikte offenbaren, dass die Einbindung Deutschlands und die Schaffung multilateraler Strukturen kein Gemeingut waren, sich gleichsam in einem Wettstreit der Ideen durchsetzen mussten. Der Marshall-Plan besaß letztlich neben seinem deutlichen multilateralen Charakter auch eine bilaterale Dimension, die sich aber erst mit Blick auf die Details offenbart.

\section{Der Schuman-Plan und die EGKS}

Beliebig konnten die USA ihre Vorstellungen aber nicht durchsetzen. Zunehmend stellten sie fest, dass ,[t]he influence of the United States on the development of the institutional

\footnotetext{
25 Hogan, Europäische Integration, S. 149.

26 Zit. n. Hogan, Europäische Integration, S. 155-156.

27 Hitchcock, The Marshall Plan, S. 158; Thomas A. Schwartz, Europäische Integration und iSpecial Relationship - Zur Durchführung des Marshall-Planes in der Bundesrepublik Deutschland, 1948- 1951, in: Deutschland und der Marshall-Plan, hrsg. v. Charles S. Maier/Günter Bischof, Baden-Baden 1992, S. 201-249, hier S. 221.

28 Schwartz, Europäische Integration, S. 203-214.
} 
aspects of European integration was negligible"29. Die Gründe dafür sind vielfältig. Entscheidend dürfte gewesen sein, dass ein zu forsches Auftreten der Vereinigten Staaten von den europäischen Partnern als Bevormundung aufgefasst werden und somit von vornherein auf Wiederstand hätte stoßen müssen. Frankreich kam in der Integration Europas eine Schlüsselrolle zu, was einmal an der unmittelbaren Nachbarschaft zu Deutschland und deren historischer Dimension lag, zum anderen verfügte kein anderer europäischer Staat über ein ähnliches politisches wie wirtschaftliches Gewicht. Frankreichs Sicherheitsinteressen wurden von den USA durchaus ernst genommen. So versicherte der neue amerikanische Außenminister Acheson seinem französischen Amtskollegen Robert Schuman, dass die USA von der französischen Regierung zwar erwarteten, "daß sie die Führung in der Integrierung von Deutschland in Europa" übernehmen müsse, stärkte inm aber zugleich mit der Zusicherung den Rücken, dass sich sein Land in diesem Falle amerikanischer Unterstützung gewiss sein könne. ${ }^{30}$ Wenig später unterbreitete Frankreich dann den Vorschlag zur Schaffung einer Montanunion, also eines supranationalen Verbandes der Stahl- und Kohleindustrien der Teilnehmerländer. Dieser sogenannte Schuman-Plan wurde schließlich am 18. April 1951 von den Außenministern Frankreichs, Deutschlands, Italiens, Belgiens, der Niederlande und Luxemburgs vertraglich verankert und erhielt mit der Europäischen Gemeinschaft für Kohle und Stahl (EGKS) seine institutionelle Form. ${ }^{31}$

Die Gründungsmitglieder der EGKS bildeten dann auch den engeren Kreis jener Länder, von denen der weitere europäische Einigungsprozess maßgeblich ausging. Großbritanniens Versuche, die Schaffung der EGKS zu torpedieren und an ihre Stelle eigene europapolitische Vorstellungen zu setzen, scheiterten am französischen Widerstand. Bereits im Sommer 1949 hatte George Kennan erkannt, dass sich Großbritannien nicht zu dem Grad integrieren ließe, wie es die USA als für Deutschland notwendig erachteten, woraufhin die amerikanische Regierung beschloss, dem Land doch eine Sonderrolle zuzugestehen. Die britische Administration konnte sich somit bei den Verhandlungen des Europarats einer stärkeren Integration widersetzen, stimmte aber der Aufnahme der, zu diesem Zeitpunkt jedoch noch nicht bestehenden, Bundesrepublik Deutschland (BRD) zu. ${ }^{32}$ Das mit einer Hinwendung zu den USA und Kanada einhergehende Ausscheren Großbritanniens bei den europäischen Integrationsbestrebungen erleichterte die französischen Position, die nicht länger mit den britischen Vorstellungen konkurrieren musste, womit das von Meinungsverschiedenheiten bis zum Stillstand reichende Spektrum an Gefahren für den Einigungsprozess zu einem gewissen Grad gebändigt war. Der SchumanPlan kam denn einerseits jenen Kräften in der US-Administration entgegen, die zwar auf Fortschritte gehofft hatten, nicht aber der Bevormundung bezichtigt werden wollten und

29 Gunther Mai, American Policy toward Germany and the Integration of Europe, 1945-1955, in: American Policy and the Reconstruction of West Germany, 1945-1955, hrsg. v. Jeffry M. Diefendorf/Axel Frohn/ Hermann-Josef Rupieder (Publications of the German Historical Institute), Cambridge 1993, S. 85-109, hier S. 85.

30 Schwartz, Europäische Integration, S. 218.

31 Wilfried Loth, Der Weg nach Europa. Geschichte der europäischen Integration 1939-1957, Göttingen $1996^{3}$, S. 85.

32 Loth, Der Weg nach Europa, S. 76-78. 
brachte andererseits die Kritiker im State Department zum Schweigen, die angesichts des schleichenden Integrationsprozesses auf stärkeren Druck ihres Landes gepocht hatten. ${ }^{33}$

Nicht zuletzt markierte der Schuman-Plan einen konkreten Schritt auf dem Weg hin zu einem wirtschaftlich geeinten Europa; eine Entwicklung, die am 25. März 1957 mit der Gründung der Europäischen Wirtschaftsgemeinschaft (EWG) einen vorläufigen Höhepunkt erreichen sollte.

\section{Die Europäische Verteidigungsgemeinschaft (EVG)}

Vor dem Hintergrund des Korea-Krieges drängten die USA neben der wirtschaftlichen auch verstärkt auf eine militärische Integration, also auf die Wiederbewaffnung Deutschlands. Dies führte zu Unruhe in Frankreich, dessen Verteidigungsminister Jules Moch und Staatspräsident Vincent Jules Auriol „erbitterte Gegner jeder Art von deutscher Wiederbewaffnung" waren. ${ }^{34}$ Nichts destotrotz dürfte auch innen klar gewesen sein, dass eine sture Blockadehaltung mehr Schaden denn Nutzen bringen konnte, bestand doch die Möglichkeit, dass die USA und Großbritannien die BRD in die NATO aufnehmen und wiederbewaffnen würden, ohne dass französische Sicherheitsinteressen Berücksichtigung fänden. ${ }^{35}$ Die Antwort Frankreichs war der Pleven-Plan, benannt nach dem ehemaligen französischen Ministerpräsidenten René Pleven, der einerseits die Aufstellung einer europäischen Armee vorsah, zugleich aber Jean Monnets Befürchtung berücksichtigte, wonach eine "Aufrüstung der Bundesrepublik vor ersten konkreten Schritten in Richtung einer europäischen Gemeinschaft”, die Chance gefährde, „ein friedliches neues Deutschland in einem befriedeten (West-) Europa zu schaffen"36. Der Pleven-Plan hatte aus der Perspektive der USA eine Reihe von Unzulänglichkeiten, zuvorderst, dass die BRD vor einem aktiven Wehrbeitrag in politische Strukturen eingebunden werden sollte, daneben, dass das Land weder über ein Verteidigungsministerium, noch über einen Generalstab, geschweige denn über eigene Truppen außerhalb der Europa-Armee verfügen sollte. Nicht zuletzt sollte die Bundesrepublik, trotz Koppelung der Europa-Armee an die NATO, kein Mitglied des Nordatlantischen Bündnisses sein. ${ }^{37}$ Der amerikanische Gegenvorschlag (Spofford Compromise) wurde demgegenüber dem Drängen der USA auf einen raschen Wehrbeitrag Deutschlands gerecht, indem er die Aufstellung deutscher Truppen auch schon vor der Schaffung gemeinsamer europäischer Institutionen vorsah. ${ }^{38}$

Nahezu zeitgleich begannen so zu Beginn des Jahres 1951 die Verhandlungen über die EVG, wie auch die Beratungen der BRD mit den alliierten Hochkommissaren über

33 Mai, American Policy, S. 97.

34 Loth, Der Weg nach Europa, S. 93.

35 David Clay Large, Grand Illusions: The United States, the Federal Republic of Germany, and the European Defense Community, 1950-1954, in: American Policy and the Reconstruction of West Germany, 19451955, hrsg. v. Jeffry M. Diefendorf/Axel Frohn/Hermann-Josef Rupieder (Publications of the German Historical Institute), Cambridge 1993, S. 375-394, hier S. 376.

36 Hans-Erich Volkmann, Adenauer, Frankreich und die Europäische Verteidigungsgemeinschaft, in: Interessen verbinden. Jean Monnet und die europäische Integration der Bundesrepublik Deutschland, hrsg. v. Andreas Wilkens (Pariser Historische Studien), Bonn 1999, S. 161-186, hier S. 169.

37 Loth, Der Weg nach Europa, S. 94.

38 Large, Grand Illusions, S. 379-380. 
einen deutschen Militärbeitrag zur NATO. Zweitere schritten relativ rasch voran, bereits im November desselben Jahres wurde ein Zwischenbericht vorgestellt, das Petersberger Abkommen, wonach weiter an einer deutschen Wiederbewaffnung im Rahmen der NATO festgehalten werde. ${ }^{39}$ Die Verhandlungen über die Europa-Armee hingegen schritten bestenfalls schleppend voran, was US-Außenminister Dean Acheson dazu veranlasste, seinen Botschafter in Paris anzuweisen, "[to] pressure the French to accept an immediate commencement of German rearmament within the NATO context". Dieser riet hiervon ab, da nur im Rahmen der EVG Frankreich und eventuelle andere Staaten „would accept the concessions to German military equality without which the Germans would not participate". Diese Einschätzung teilte auch der Oberkommandierende der Alliierten Streitkräfte, Dwight D. Eisenhower. ${ }^{40}$ Die letztliche Entschlossenheit der USA, die militärische Einbindung Deutschlands im europäischen Rahmen zu suchen, zwang beide Seiten zu Zugeständnissen und führte schließlich am 27. Mai 1952 zu einer offiziellen Einigung, die nunmehr lediglich der Ratifikation durch die nationalen Parlamente bedurfte.

Frankreichs Engagement für die EVG erklärte sich vor allem aus dem Interesse an einer gemeinsamen Verteidigung Europas an der Seite der USA. Als Eisenhower Anfang 1953 amerikanischer Präsident und John Foster Dulles sein Außenminister wurde, waren sie bestrebt, die amerikanische Außenpolitik ökonomisch auf stabilere Beine zu stellen, was in Dulles folgender Aussage beredten Ausdruck findet: "If economic stability goes down the drain, everything goes down the drain." 41 Konkret waren damit der Ausbau der strategischen Nuklearkapazitäten der USA, vor allem aber Überlegungen über einen Truppenabbau in Europa verknüpft. Dies schürte in Frankreich den Verdacht, dass die EVG nicht als Beitrag zu einer gemeinsamen Verteidigung Europas gedacht war, sondern dass europäische Streitkräfte amerikanische Truppen auf dem Kontinent ersetzen sollten, wodurch das Land "sicherheitspolitisch [...] vollständig in die Abhängigkeit von den USA zu geraten" befürchtete. ${ }^{42}$ Frankreichs Vorbehalte kamen schließlich in der Ablehnung der EVG durch die französische Nationalversammlung am 30. August 1954 zum Ausdruck.

Im Ringen um die EVG offenbarte sich das prinzipielle Bestreben sowohl der Truman-, wie auch der Eisenhower-Administration, eine multilaterale Lösung zu finden, wobei die militärische Einbindung Deutschlands auf europäischer Ebene zeitweise in den Schatten einer grundsätzlichen militärischen Einbindung der Bundesrepublik trat.

\section{Die North Atlantic Treaty Organization (NATO)}

Die EVG war keineswegs die erste europäische Initiative hin zur Schaffung eines militärischen Bündnisses. Bereits am 17. März 1948 war es zum Brüsseler Pakt gekommen, dem neben Frankreich und Großbritannien auch die Benelux-Staaten angehörten, und der sich nicht nur gegen die Sowjetunion richtete, sondern zugleich vorsah, "to take such measures as

39 Large, Grand Illusions, S. 381

40 Zit. n. Large, Grand Illusions, S. 382-383.

41 Zit. n. Thomas U. Schöttli, USA und EVG. Truman, Eisenhower und die Europa-Armee (Europäische Hochschulschriften), Bern 1994, S. 321.

42 Loth, Der Weg nach Europa, S. 106-107. 
may be necessary in the event of a renewal by Germany of a policy of aggression"43. Der Brüsseler Pakt war also ein Instrument der doppelten Eindämmung, faktisch aber wenig mehr als ein Papiertiger, da es sowohl an militärischen wie wirtschaftlichen Mitteln fehlte, um inm eine konkete Form zu geben. ${ }^{44}$ Seine Bedeutung lag mehr im symbolischen Gehalt, vermittelte das Abkommen den USA doch, dass es in Europa Bestrebungen zur Schaffung eines gemeinsamen Sicherheitsbündnisses gab. Frankreich und Großbritannien ging es dabei, wie bereits weiter oben kurz angedeutet, vor allem darum, „to involve the United States militarily in Europe on a permanent basis" 45 . Dass sich die USA letztlich darauf einließen, sich in Europa dauerhaft („permanent[ly]") zu involvieren, kam einem radikalen Bruch mit dem im zweiten Kapitel beschriebenen Grundsatz der USAußenpolitik gleich, sich nicht auf "entangling alliances" einzulassen. Zunehmend trieben die Vereinigten Staaten selbst die Schaffung gemeinsamer Sicherheitsstrukturen voran, was ihre Entschlossenheit, einen Beitrag zur Stabilisierung Europas zu leisten, noch weiter verdeutlicht. ${ }^{46}$

Die im Juni 1948 vom Kongress verabschiedete Vandenberg-Resolution war ein entscheidender Schritt der USA in der Abwendung vom Konzept der "entangling alliances” und in vielfacher Weise von Bedeutung. Die Vandenberg-Resolution „authorized the United States to enter into mutual defense agreements only with countries capable of providing self-defense and contributing to the security of the United States" ${ }^{47}$. Sie verhinderte also einerseits, dass die USA in eine Allianz hineingezogen würden, in der das Land die Hauptlasten zu tragen hätte, bildete aber im gleichen Zug die politische und verfassungsrechtliche Basis für ein transatlantisches Bündnis. Der Senat hatte mit dem Beschluss seine Eigenständigkeit in außenpolitischen Fragen unter Beweis gestellt und bewusst oder auch nicht, französischem Drängen einen Riegel vorgeschoben, „die Angloamerikaner [...] mit verbindlichen Abreden langfristig in Deutschland festzunageln"48. Des Weiteren wurde das Recht des Kongresses, Krieg zu erklären, gestärkt. Ein Recht, das als ,juristischer Stolperstein [...] schon nach dem ersten Weltkrieg den Beitritt der Vereinigten Staaten zum Völkerbund verhindert hatte"49.

Auch den in Paris geäußerten Sicherheitsinteressen galt es von amerikanischer Seite gerecht zu werden. Bisweilen schien es aber fast den Anschein zu machen, als ob Frankreich mehr Angst vor Deutschland, denn vor der Sowjetunion hatte, was Außenminister Marshall

43 Zit. n. Edward Fursdon, The Role of the European Defense Community in European Integration, in: NATO: The Founding of the Atlantic Alliance and the Integration of Europe, hrsg. v. Francis H. Heller/John R. Gillingham, Basingstoke-London 1992, S. 213-240, hier S. 215.

44 Fursdon, The Role of the European Defense Community, S. 215-216.

45 Irwin M. Wall, France and the North Atlantic Alliance, in: NATO: The Founding of the Atlantic Alliance and the Integration of Europe, hrsg. v. Francis H. Heller/John R. Gillingham, Basingstoke-London 1992, S. 45-56, hier S. 46.

46 Wall, France and the North Atlantic Alliance, S. 46.

47 Sayuri Guthrie-Shimizu, Japan, the United States, and the Cold War, 1945-1960, in: The Cambridge History of the Cold War I, hrsg. Melvyn P. Leffler/Odd Arne Westad, Cambridge 2010, S. 244-265, hier S. 264.

48 Gero von Gersdorff, Die Gründung der Nordatlantischen Allianz (Entstehung und Probleme des Atlantischen Bündnisses), München 2009, S. 168.

49 Klaus Schwabe, Bündnispolitik und Integration 1949-1956, in: Das Nordatlantische Bündnis 1949-1956, hrsg. v. Klaus A. Maier/Norbert Wiggershaus (Beiträge zur Militärgeschichte), München 1993, S. 71-87, hier S. 75-76. 
schließlich zu der Bemerkung verleitete, "dass es angesichts der Berichte über die politisch instabilen Regierungen in Frankreich und Zweifeln an deren Standhaftigkeit in Berlin eigentlich an der US-Regierung sei, von Frankreich Zusicherungen der Zuverlässigkeit einzufordern"50. Diese Aussage bringt einerseits die hohe Bedeutung Deutschlands bei führenden amerikanischen Politikern zum Ausdruck, liefert aber auch zugleich Zeugnis von Frankreichs Bedeutung ab, denn eine innere Schwäche des Landes musste es in den Augen der USA anfällig für kommunistische Tendenzen machen, was es unter allen Umständen zu vermeiden galt. Im abschließenden Vertrag vom 4. April 1949 gestanden die Vereinigten Staaten Frankreich zwar keine „automatische[n] militärische[n] Hilfszusage[n], dafür aber doch ein automatisches allgemeines Beistandsversprechen" zu, das auch militärische Maßnahmen miteinschließen konnte. ${ }^{51}$ Das Entgegenkommen der USA und der letztliche rasche Abschluss der NATO-Verhandlungen mag zu einem nicht geringen Teil von der Wahrnehmung einer äußeren Bedrohung durch die Sowjetunion beeinflusst worden sein, hatten doch im Februar 1948 tschechoslowakische Kommunisten mit der Rückendeckung Moskaus die dortige, demokratisch legitimierte Regierung von der Macht geputscht. Verstärkt wurden diese Wahrnehmungen dann noch durch den sowjetischen Versuch, Westberlin mittels einer Blockade von der Außenwelt abzuschneiden. ${ }^{52}$

Ein deutscher NATO-Beitritt wäre im Frühjahr 1949 trotz allem noch undenkbar gewesen und kam denn auch erst nach dem Scheitern der EVG „im Mai 1955 durch die sogenannte NATO/WEU-Lösung" zustande. ${ }^{53}$ Die Westeuropäische Union (WEU) war ein auf dem Brüsseler Pakt fußendes Militärbündnis. Damit war die BRD sowohl in europäische, wie auch atlantische multilaterale Bündnisstrukturen erfolgreich eingebunden worden.

\section{Wandel der Außenpolitik unter George W. Bush}

\section{Außenpolitische Überzeugungen zur Rolle der USA in der Welt}

Bei George W. Bushs Amtsantritt im Januar 2001 deutete nichts auf einen Wandel der US-Außenpolitik hin, setzte sich sein außenpolitisches Team doch aus Leuten zusammen, die bereits in vorhergehenden Administration, unter anderem jener seines Vaters, George H. W. Bush, tätig gewesen waren and "[who] were thought to care about great power diplomacy, not moral crusades"54. Derartige Hoffnungen dürfte auch der deutsche Bundeskanzler Gerhard Schröder gehegt haben, als er zu seinem Antrittsbesuch ins Weiße Haus aufbrach. Bereits im Anflug auf Washington befindlich, musste er dann aber hören, dass Präsident Bush „mit größter Selbstverständlichkeit verkündete, Amerika [...] werde das Klimaschutzabkommen [von Kyoto] natürlich nicht unterzeichnen"55. Diese Entscheidung

50 Von Gersdorff, Die Gründung der Nordatlantischen Allianz, S. 173.

51 Schwabe, Bündnispolitik und Integration, S. 77.

52 Gaddis, Der Kalte Krieg, S. 48-50.

53 Klaus Larres, Eisenhower, Dulles und Adenauer. Bündnis des Vertrauens oder Allianz des Mißtrauens?. (1953-1961), in: Deutschland und die USA im 20. Jahrhundert. Geschichte der politischen Beziehungen, hrsg. v. Klaus Larres/Torsten Oppelland, Darmstadt 1997, S. 119-151, hier S. 133.

55 Claus Kleber, Amerikas Kreuzzüge. Was die Weltmacht treibt, München 2005, S. 69 
des Präsidenten spiegelt ein außenpolitisches Denken wider, das zwar seit einigen Jahren in gewissen politischen Kreisen zirkuliert war, bis dahin aber kaum bis wenig Einfluss auf die praktische US-Außenpolitik gehabt hatte.

Stephen F. Szabo teilt die außenpolitischen Berater George Bushs in drei Kategorien ein. Die „traditional realists" sind Vertreter des Multilateralismus in dem Sinne, "that they see the need for alliances and a broader international framework - which minimize the dangers of overextension and the formation of countervailing coalition - in order to best exercise power"56. Der einzige Vertreter dieser Schule in Bushs Mannschaft war Außenminister Colin Powell. Der überzeugteste Exponent der Neokonservativen, die Szabo als "hyper-Wilsonian in their belief that the world can be democratized and thus pacified" charakterisiert, war der stellvertretende Verteidigungsminister Paul Wolfowitz. ${ }^{57}$ Die letzte Kategorie bilden die "Nationalist Conservatives", „[who are] deeply pessimistic about the threats the rest of the world poses to American security and values" und folglich nicht danach trachten "to remake the world" sondern "simply strive to protect America" ${ }^{\text {" }}$. Zu dieser Kategorie rechnet Szabo den Präsidenten selbst, Vizepräsident Richard Cheney und Verteidigungsminister Donald Rumsfeld. Die Nationale Sicherheitsberaterin Condoleezza Rice kam ursprünglich aus der Schule der Traditional Realists, fand aber unter Bush ihren Weg ins Bündnis von Neokonservativen und Nationalist Conservatives.

Es war Cheney, der dieses Bündnis der Konservativen zusammenbrachte, was nach einem Blick auf die „Defense Planning Guidance” von 1992 nicht weiter verwundert. Von Zalmay Khalilzad verfasst, von Paul Wolfowitz als Unterstaatssekretär im Verteidigungsministerium in Auftrag gegeben und von Verteidigungsminister Cheney gegenüber Khalilzad mit den Worten bedacht, "You've discovered a new rationale for our role in the world"59, wurde in diesem Dokument vorgeschlagen "that the United States should work actively to block the emergence of any potential competitor to American power", was vor allem mittels „unmatchable military strength" bewerkstelligt werden sollte. So sollten die USA zwar "the goal of European integration" unterstützen, „[but] must seek to prevent the emergence of European-only security arrangements which would undermine NATO"60. Man kann diesem Bekenntnis jedwede Glaubwürdigkeit absprechen, denn im selben Dokument heißt es auch, "[that the] first objective is to prevent the re-emergence of a new rival" 6 l. Prinzipiell könnten so alle europäischen Einigungsbestrebungen, gleich ob politischer, wirtschaftlicher oder militärischer Natur von den USA als rivalisierende Bestrebungen gedeutet werden, die es im Keim zu ersticken gälte. Zwar relativierte eine wenige Monate später erschienene Überarbeitung des Dokuments diesen Punkt, wonach es lediglich das Ziel der Vereinigten Staaten sei „to preclude any hostile power from dominating a region

\footnotetext{
56 Szabo, Parting Ways, S. 53-54.

57 Ebd., S. 55

58 Ebd., S. 61

59 Zit. n. Mann, Rise of the Vulcans, S. 2011.

60 The New York Times, Excerpts From Pentagon's Plan: 'Prevent the Re-Emergence of a New Rival', 08.03.1992, [http://www.nytimes.com/1992/03/08/world/excerpts-from-pentagon-s-plan-prevent-there-em ergence-of-a-new-rival.html?pagewanted=all\&src=pm], eingesehen 10.09.2013. 
critical to our interests" 62 , doch sind die Abänderungen überwiegend kosmetischer Natur. Beide Dokumente durchzieht als roter Faden ein übermächtiges Amerika, das gleichsam so stark ist, dass anderen von vornherein jeder Anreiz zu konkurrieren genommen sein soll.

Unter dem Strich gab es Differenzen im Denken von Bushs Beratern, doch "[a]ll of them believed in the importance of American military power"63.

\section{9/11 und der Krieg gegen den Terrorismus}

Im Sommer 2001 befanden sich die Vereinigten Staaten auf dem Höhepunkt ihrer politischen, militärischen und wirtschaftlichen Macht. Entsprechend groß war der Schock, als mit dem Pentagon und den beiden Türmen des World Trade Center am 11 . September 2001 zwei bedeutende Symbole ihrer Macht beschädigt bzw. ganz zerstört wurden. Pearl-Harbor-gleich wurden die Zentren amerikanischer Militär- und Wirtschaftsmacht getroffen und das Land stand unter dem Eindruck, "[that] the world was going bad rapidly. We had to do something. We had the power to do it by ourselves. We didn't have time to consult our friends and allies. We could do it alone"64. So formulierte es Brent Scowcroft, Nationaler Sicherheitsberater und George H. W. Bush. Im Unterschied zu Pearl Harbor ging dieser Angriff aber nicht von einem Staat, sondern von einer Organisation aus und nicht nur dieser, sondern dem ganzen Phänomen, für das sie stand, wurde der Krieg erklärt. Dieses Phänomen hieß Terrorismus. In einer Ansprache am Abend des 11. September verkündete Präsident Bush, dass nicht unterschieden werde, „zwischen denen, die diese Taten geplant haben, und denen, die sie beherbergen"65. Damit standen sowohl Osama Bin Laden und seine Terrororganisation Al-Oaida, die die Anschläge geplant, als auch Afghanistan, das diesen Unterschlupf gewährt hatte, im Fadenkreuz der Vereinigten Staaten.

Internationale Rückendeckung erhielten die USA durch eine am 12. September verabschiedete Resolution des Sicherheitsrats der Vereinten Nationen, welche die Anschläge vom Vortag verurteilte und "call[ed] on all States to work together urgently to bring to justice the perpetrators, organizers and sponsors of theses terrorist attacks"66. Mit der am selben Tag, und bis dato einmaligen Ausrufung des Bündnisfalls durch die NATO, bekundeten deren Mitglieder ihre Bereitschaft, "shoulder to shoulder"67 mit den USA in den Krieg gegen den Terrorismus zu ziehen. Doch die Reaktionen innerhalb der US-Regierung waren zwiespältig. Während Colin Powell und Condoleezza Rice darauf drängten, „so viele [NATO-Partner] wie möglich [...] auch mit militärischen Kräften einzubinden", bremste Verteidigungsminister Rumsfeld solche Vorschläge mit der Begründung, ,[d]ie Koalition

62 Information Clearing House, Defense Strategy for the 1990s: The Regional Defense Strategy, Januar 1993, [http://www.informationclearinghouse.info/pdf/naarpr_Defense.pdf], eingesehen 10.09.2013, S. 4.

63 Mann, Rise of the Vulcans, S. xvi.

64 Zbigniew Brzezinski/Brent Scowcroft, America and the World. Conversations on the Future of American Foreign Policy, New York 2008, S. 18.

65 Bob Woodward, Bush at War. Amerika im Krieg, Stuttgart-München 2003, S. 45.

66 United Nations Security Council, Resolution 1368 (2001), 12.09.2001, [http://www.un.org/en/ga/ search/view_doc.asp?symbol=S/RES/1368\%282001\%29], eingesehen 13.09.2013.

67 Tony Blair, A Journey, London 2011, S. 352. 
[...] [müsse] dem Konflikt angepaßt sein"68. Die Anfrage der US-Regierung an die NATO um Unterstützung war letztlich ein Kompromiss, um Powell und Rice zufriedenzustellen und die Verbündeten nicht allzu sehr vor den Kopf zu stoßen. Bushs Siebenpunkteprogramm sah denn auch nur in zwei Fällen "den Einsatz gemeinsamer NATO-Ressourcen” vor und setzte ansonsten auf bilaterale Beistandsmaßnahmen, wie Überflugrechte. ${ }^{69}$ Damit wurde das NATO-Bündnis aus Sicht so mancher Mitglieder vom Sicherheitsbündnis weitgehend gleichberechtigter Partner zum „Dienstleistungsunternehmen für amerikanisch geführte Militäroperationen $[\ldots]^{\prime \prime 7}$.

Am 7. Oktober 2001 liefen dann die amerikanischen Luftangriffe der Operation Enduring Freedom (OEF) an, lediglich von Großbritannien unterstützt. Das Streben der USA nach Handlungsfreiheit war unübersehbar. Doch trotz Bushs wenig taktvollem Agieren, erhielt er doch die Unterstützung der NATO-Partner. Kanzler Gerhard Schröder sicherte den USA „uneingeschränkte Solidarität" ${ }^{\text {"1 }}$ zu und sein Land partizipierte, abgesehen von Kampfeinsätzen, „am gesamten militärischen Spektrum"72. Darüber hinaus erklärte die BRD ihre Bereitschaft zur Beteiligung an einer Schutztruppe, forderte allerdings eine dahingehende UN-Resolution und die Trennung vom OEF-Mandat. ${ }^{73}$ Die am 20. Dezember verabschiedete Resolution segnete dann "the establishment [...] of an International Security Assistance Force"74 (ISAF) ab. Zum 1. August 2013 umfasste das deutsche ISAF-Kontingent 4400 Personen und war damit das drittgrößte nach jenem der USA und Großbritanniens. ${ }^{75}$

\section{Der Irak und das angespannte Verhältnis zu den Verbündeten}

Der Umgang der US-Regierung mit den Verbündeten bezüglich Afghanistan war ein erster Vorgeschmack auf ihr Verhalten im Falle des Irak. Die Spannungen zwischen den USA und den Bündnispartnern offenbarten eine tiefe Kluft, wie sie auch das außenpolitische Establishment der Vereinigten Staaten selbst aufwies.

In seiner Rede zur Lage der Nation vom 29. Januar 2002 verkündete Präsident Bush, "[that] [o]ur goal is to prevent regimes that sponsor terror from threatening America [...] with weapons of mass destruction", wobei Nordkorea, der Iran und der Irak die von inm betitelte "axis of evil" bildeten. ${ }^{76}$ Je nach situativer Argumentation lässt sich so

68 Woodward, Bush at War, S. 202

69 Marco Overhaus, Die deutsche NATO-Politik. Vom Ende des Kalten Krieges bis zum Kampf gegen den Terrorismus (Außenpolitik und Internationale Ordnung), Baden-Baden 2009, S. 262.

70 Klaus-Dieter Schwarz, Die NATO ist tot - es lebe die NATO, in: Die Welt, 14.5.2002, zit. n. Overhaus (2009), S. 263.

71 Zit. n. Jürgen Hogrefe, Gerhard Schröder. Ein Porträt, Berlin 2002, S. 210.

72 Overhaus, Die deutsche NATO-Politik, S. 265.

73 Ebd., S. 269-270.

74 United Nations Security Council, Resolution 1368 (2001), 12.09.2001,[http://mww.un.org/en/ga/ search/v iew_doc.asp?symbol=S/RES/1368\%282001\%29], eingesehen 13.09.2013.

75 International Security Assistance Force, Troop Numbers and Contributions, 01.08.2013, [http://www. isaf .nato.int/images/stories/File/Placemats/2013-08-01\%20ISAF\%20Placemat-final.pdf], eingesehen 14.09.2013.

76 U.S. Government Printing Office, Compilation of Presidential Documents, Addresses and Remarks, Address Before a Joint Session of the Congress on the State of the Union, 29.01.2002, S. 135, [http://www.gpo. gov/fdsys/pkg/WCPD-2002-02-04/pdf/WCPD-2002-02-04-Pg133-3.pdf], eingesehen 13.09.2013. 
das Vorgehen gegen Staaten, die nach Massenvernichtungswaffen streben, als Teil des weltweiten Kampfs gegen den Terror stilisieren. Dass in erster Linie der Irak im Fokus der US-Administration stand, legt ein wenige Tage später in der New York Times erschienener, sich auf Colin Powell stützender Artikel nahe, wonach „the administration was considering [...] options to topple Saddam Hussein" und Powell mit den Worten zitiert, dass "with respect to Iran and [...] North Korea, there is no plan to start a war"77.

Was bei der Lektüre von Bushs Rede verwundert ist, dass er außer Pakistan keinem anderen Land für die den USA gewährte Unterstützung dankte. Mehr noch, er versicherte den im Angesicht des Terrorismus Zögerlichen („timid") "[that] [i]f they do not act, America will"78. Zwar wies der Präsident in einem Interview vom 20. August 2002 den Vorwurf, er sei ein Unilateralist, als "Ressentiment" von sich. ${ }^{79}$ Die europäische Wahrnehmung war offenbar eine andere, denn bereits im Monat zuvor hatte der britische Außenminister Jack Straw gegenüber Premierminister Tony Blair seinen Eindruck bekundet, wonach „,'military action was now seen as inevitable' by Washington"80.

In der im September 2002 veröffentlichten "National Security Strategy" offenbart sich ein Wandel weg von festen Bündnisstrukturen hin zu "mission-based coalitions", die "as broad as practicable"81 organisiert sein sollten und auch als "[c]oalitions of the willing"82 bezeichnet werden. Darüber hinaus klingt eine Art Missionsstreben zur Verbreitung amerikanischer Werte an, das in der Erklärung Ausdruck findet, der Krieg gegen den Terror sei letztlich auch ein Kampf "for [...] democratic values and [the American] way of life"83. Die National Security Strategy von 2002 kann also durchaus als ein strategischvisionäres, von idealistischen Vorstellungen durchzogenes Dokument gesehen werden, das für taktische Erwägungen eines Colin Powell keinen Raum ließ, in dessen Augen "[s] preading ,freedom' around the world was not much of a game plan, no matter how much firepower you had"84.

Bushs Appell an die Vereinten Nationen vom 12. September 2002, die gegen den Irak bereits beschlossenen Sanktionen mit Nachdruck durchzusetzen ${ }^{85}$, scheint nur auf den ersten Blick Powells Multilateralismus entgegenzukommen, denn bevor die UN eine dahingehende Resolution verabschieden konnten, hatte die US-Regierung bereits die Erlaubnis des Kongresses eingeholt „to use the Armed Forces [to] defend the national

77 Zit. n. Michael R. Gordon/David E. Sanger, A Nation Challenged: Iraq; Powell Says U.S. Is Weighing Ways To Topple Saddam Hussein, 13.02.2002, [http://www.nytimes.com/2002/02/13/world/a-nationchallenged-iraq-powell-says-us-is-weighing-ways-to-topple-hussein.html?pagewanted=2], eingesehen 17.09.2013.

78 Address Before a Joint Session of the Congress on the State of the Union, 29.01.2002, S. 135.

79 Woodward, Bush at War, S. 376.

80 Karen DeYoung, Soldier. The Life of Colin Powell, New York 2006, S. 405.

81 National Security Strategy Archive, The National Security Strategy of the United States of America, S. 25, [http://nssarchive.us/NSSR/2002.pdf], eingesehen 09.09.2013.

82 National Security Strategy Archive, The National Security Strategy, Vorwort des Präsidenten.

83 Ebd., S. 7.

84 DeYoung, Soldier, S. 403.

85 C-Span Video Library, U.S. President Speech, 12.09.2002, [http://www.c-spanvideo.org/program/17261 1-2], eingesehen 20.09.2013. 
security [...] against [...] Iraq"86. Dieses Gesetz des amerikanischen Kongresses und die Aussage des britischen Außenministers vom Juli legen nahe, dass der Entschluss zum Krieg gegen den Irak schon frühzeitig gefällt worden war und nunmehr lediglich legitimierende Gründe gesucht werden mussten. Auch die deutsche Justizministerin Herta DäublerGmelin mochte Mitte September bereits einen solchen Eindruck gehabt haben, als sie mitten im Wahlkampf George W. Bushs Methoden mit denen Adolf Hitlers verglich, was ein vorläufiges Tief in den amerikanisch-deutschen Beziehungen markierte. ${ }^{87}$

Am 5. Februar 2003 präsentierte dann Außenminister Powell vor der UN von den USA selbst gesammelte Beweise, wonach der Irak über Massenvernichtungswaffen verfüge, ungeachtet des Umstands, dass kurz zuvor der Leiter der Internationalen Atomenergiebehörde (IAEA) Mohamed ElBaradei festgestellt hatte, "[that] we have to date found no evidence that Iraq has revived its nuclear weapons programme"88. Powells Anschluss an die Kriegsbefürworter lag paradoxerweise an Frankreich und Deutschland, von Rumsfeld als "old Europe"89 (de-)klassifiziert, die mit ihrer Blockadehaltung, "[that] nothing justifies [...] military action"90, dem multilateralen Ansatz des Außenministers die Grundlage entzogen hatten.

\section{Die Administration Barack Obamas}

\section{Afghanistan und die Finanzkrise}

Barack Obamas Wahlkampfslogan „Yes, we can"91 spiegelt einen Tatendrang wider, der für die US-Außenpolitik angesichts der zahlreichen Herausforderungen bitter notwendig war. Der ebenfalls mitschwingende Idealismus ließ sich aber nicht ohne weiteres in politische Realitäten gießen. Zu groß waren die Probleme, die von den Kriegen im Irak und Afghanistan, über die belasteten Beziehungen zu zahlreichen, ehemals engen Verbündeten bis hin zum teils mehr, teils minder schwelenden Nahostkonflikt und Irans Atomprogramm reichten. Ähnlich wie bei Bush schon binnen weniger Monate nach Amtsantritt Obamas von einer Revolution in der US-Außenpolitik zu sprechen, wäre verfehlt. Der Afghanistan- und Irakkrieg waren nicht von heute auf morgen zu beenden und so konnte Obama lediglich in kurz- und mittelfristiger Hinsicht die Akzente verschieben, was etwa in seiner Erklärung vom 27. Februar 2009 Ausdruck findet, dass zum 31 . August 2010 ,[the] combat mission in Iraq will end"92.

86 U.S. Government Printing Office, Public and Private Laws, Authorization for Use of Military Force Against Iraq Resolution of 2002, 16.10.2002, [http://www.gpo.gov/fdsys/pkg/PLAW-107pub/243/pdf/PLA W-107publ243.pdf], eingesehen 20.09.2013.

87 Szabo, Parting Ways, S. 29.

88 United Nations, The Status of Nuclear Inspections in Iraq. Statement to the United Nations Security Council, 27.01.2003, [http://www.un.org/news/dh/iraq/elbaradei27jan03.htm], eingesehen 20.09.2013.

89 Zit. n. Szabo, Parting Ways, S. 39.

90 DeYoung, Soldier, S. 434.

91 CNN, Obama speech: ,Yes, we can change', 27.01.2008, [http://edition.cnn.com/2008/POLITICS/01/26/ obama.transcript/], eingesehen 06.10.2013.

92 The New York Times, Obama's Speech at Camp Lejeune, N.C., 27.02.2009, [http://www.nytimes.com/2 009/02/27/us/politics/27obama-text.html?pagewanted=1\&_r=0], eingesehen 06.10.2013. 
Obwohl Obama als Senator 2007 noch gegen Truppenerhöhungen im Irak gestimmt hatte $^{93}$, ging er als Präsident im Falle Afghanistans genau diesen, vom dortigen Oberkommandierenden David Petraeus vertretenen Weg. Damit machte er sich dessen Strategie der "counterinsurgency" zu eigen, die nach Robert F. Kennedy darauf abzielte, "[to] bring the insurgent back into the national life"94. Präsident Obama griff damit auch zu einem gewissen Grad auf Überlegungen Colin Powells und von Reagans Verteidigungsminister Caspar Weinberger zurück. Die sogenannte Weinberger Doctrine bestand auf einer genauen Analyse der Kriegsziele, denen dann die Truppenzahl angepasst sein sollte. ${ }^{95}$ Nach Powell sollte daneben vor Kriegsausbruch bereits eine "exit strategy" feststehen. ${ }^{96}$ Der Golfkrieg von 1990/1991 war ein Paradebeispiel für die Ausführung einer Militäraktion nach der Weinberger und Powell Doctrine, gelang es doch binnen sechs Monaten mit einer "coalition of major powers"97 die irakische Armee aus Kuwait zu vertreiben. Nach Erreichen dieses Kriegsziels wäre ein Sturz Saddam Husseins theoretisch ein Leichtes gewesen, doch wurde ganz im Einklang mit den Überlegungen des damals höchsten amerikanischen Generals, Colin Powell, und aus Rücksicht auf die arabischen Verbündeten und die unabsehbaren Folgen für die Region darauf verzichtet. Powells ursprünglicher Widerstand gegen die Irak-Politik des jüngeren Bush hatte sich demnach wesentlich aus der Nichterfüllung zahlreicher Punkte seiner Kriterienliste gespeist.

Da sich die Counterinsurgency-Strategie im Irak auszuzahlen schien (seit ihrer Umsetzung Anfang 2007 waren die Verlustzahlen der USA binnen eines Jahres auf ihr niedrigstes Niveau seit Kriegsbeginn gefallen ${ }^{98}$ ), wagte Obama das Konzept auf Afghanistan zu übertragen. Die konkrete Implementierung oblag Verteidigungsminister Robert Gates, der diesen Posten auch unter Bush seit 2006 bekleidet hatte und eben David Petraeus. Counterinsurgency setzte zwei Dinge voraus: mehr Truppen und mehr Zeit. Der Präsident war zwar willens, mehr Truppen zu entsenden, aber nicht auf unbegrenzte Zeit. So verkündete Obama Ende 2009 in einem ersten Schritt 30.000 zusätzliche Truppen nach Afghanistan abzukommandieren, die ersten Truppenteile aber im Sommer 2011 zurückzuführen. In derselben Rede würdigte Obama die Freunde der USA, „[who] fought and bled and died alongside us", betonte aber auch, dass das Engagement in Afghanistan ein internationales Anliegen sei, weshalb er auch die Verbündeten um

93 United States Senate, U.S. Senate Roll Call Votes $110^{\text {th }}$ Congress $-1^{\text {st }}$ Session. A bill to express the sense of Congress on Iraq, 17.02.2007, [http://www.senate.gov/legislative/LIS/roll_call_lists/roll_call_vote_cfm.cf $m$ ?congress $=110$ \&session= 1 \&vote=00051 ], eingesehen 23.09.2013.

94 Zit. n. James Mann, The Obamians. The Struggle Inside the White House to Redefine American Power, New York 2012, S. 122.

95 Walter LaFeber, The Rise and Fall of Colin Powell and the Powell Doctrine, in: Political Science Quarterly 124 (2009), Nr. 1, S. 71-93, hier S. 73, [http://onlinelibrary.wiley.com/doi/10.1002/j. 1538-165X.2009. tb00642.x/abstract], eingesehen 29.08.2013.

96 Zit. n. LaFeber, The Rise and Fall, S. 76

97 LaFeber, The Rise and Fall, S. 75.

98 Mann, The Obamians, S. 8. 
Truppenerhöhungen gebeten habe. ${ }^{99}$ Die BRD für ihren Teil antwortete darauf mit einer „Erhöhung der Mandatsobergrenze" von nahezu 19 Prozent. ${ }^{100}$

Diese Gesten waren noch keine Rückkehr zum multilateralen Internationalismus, müssen vielmehr im historischen Gesamtkontext gesehen werden. Zum Amtsantritt Obamas Anfang 2009 befanden sich die USA in der größten Finanz- und Wirtschaftskrise seit 1929, die sich, vom Kollaps der amerikanischen Großbank Lehman Brothers ausgelöst, rasch weltweit ausgebreitet hatte. Der globale Charakter dieser Krise überzeugte die Obama-Administration, dass Lösungen nur im globalen Rahmen gefunden werden konnten, weshalb der Präsident überwiegend die Gipfeltreffen der G20 als „premium forum for international cooperation"101 nutzte. Im Gegensatz zu den G7 war bei den G20 auch China vertreten, das aufgrund seines wirtschaftlichen und politischen Gewichts nicht länger ignoriert werden konnte. Dieser Wandel weg von den G7 hin zu den G20 "reflected [therefore] the changing global distribution of economic power"102.

Unter Obama verschob sich der amerikanische Fokus zunehmend von Europa auf Asien und besonders China. Unter Wahrung dieser globalen Perspektive bekommen auch die Bestrebungen der USA zusammen mit Europa „die größte Freihandelszone der Welt zu schaffen"103, eine neue Qualität, ließen sie sich doch dahingehend interpretieren, dass ein Wirtschaftsblock gegen China gebildet werden sollte.

\section{Die Arabische Revolution und Libyen}

Infolge der Selbstverbrennung des 26-jährigen Mohammed Bouazizi Mitte Dezember 2010 breitete sich eine Welle des Protests über ganz Tunesien aus, das während der Herrschaft des Diktators Zine al-Abidine Ben Ali jahrelang unter „politischer Repression, endemischer Korruption, sozialen Umbrüchen, Armut" gelitten hatte. Die Unruhen führten binnen eines Monats zum Sturz Ben Alis. ${ }^{104}$ Es war der Auftakt der Arabischen Revolution. Aus US-Perspektive kam diesen Ereignissen zunächst kaum Bedeutung zu, andernfalls hätte Außenministerin Hillary Clinton Ende Januar 2011 nicht erklärt "that the Egyptian government is stable"105. Doch auch Äypten, dieser Pfeiler der amerikanischen Nordafrikaund Nahostpolitik, wurde von Unruhen heimgesucht und bereits am 11. Februar 2011 trat Staatschef Hosni Mubarak zurück.

99 The White House, Remarks by the President in Address to the Nation on the Way Forward in Afghanistan and Pakistan, 01.12.2009, [http://www.whitehouse.gov/the-press-office/remarks-presidentaddress-nation-way-forward-afghanistan-and-pakistan], eingesehen 07.10.2013.

100 Bundeswehr, Chronologie des Einsatzes in Afghanistan ISAF, 04. 10.2013, [http://www. einsatz. bundes wehr. de/portal/a/einsatzbw/lut/p/c4/LcgxDoAgDEbhs3gBurt5C3Uxv1 qhgRQjVRJOr4N50_dopi_FIx4mWZF opGmTfql urTsvLFpg7SOi3ZzSv9gaOxw-OKUY1G3hypoXKTjojEP3AjH-WWO!/], eingesehen 07.10.2013.

101 G20, What is the G20, o.D., [http://www.g20.org/docs/about/about_G20.html], eingesehen 02.10.2013.

102 Martin S. Indyk/Kenneth G. Lieberthal/Michael E. O'Hanlon, Bending History. Barack Obama's Foreign Policy, Washington D.C. 2012, S. 31.

103 Cai Rienäcker, Freier Handel zwischen zwei Kontinenten, 13.02.2013, [http://www.tagesschau.de/wirts chaft/freihandelszone 102.html], eingesehen 09.10.2013.

104 Michael Lüders, Tage des Zorns. Die Arabische Revolution verändert die Welt, München 2011, S. 17-18.

105 Indyk/Lieberthal/O’Hanlon, Bending History, S. 142. 
Obamas Agieren angesichts der Unruhen war inkohärent. Von einem realistischen Standpunkt aus musste es die USA nicht interessieren, wie die Staatschefs ihre Länder regierten. Mubarak, "[who had] supported American positions in the Middle East and peaceful relations with Israel" war während seiner fast dreißigjährigen Herrschaft ein Stabilitätsanker für die Region gewesen, was den USA genügt hatte. ${ }^{106}$ Obama legte diesem zwar letztlich den Rücktritt nahe, doch sah die Sache bei anderen arabischen Staaten schon wieder ganz anders aus. Als die Unruhen im Königreich Bahrain mithilfe des saudischen Militärs erstickt wurden, hielt sich Obama weitestgehend bedeckt. Charakteristikum seiner Außenpolitik gegenüber Ländern, in denen es zu Ausschreitungen kam, war also keine „policy for democracy for everyone, but rather by circumstance [...] depending on American interests"107.

Der amerikanische Präsident scheute zwar nicht pauschal vor militärischen Aktionen zurück, was die Truppenerhöhungen in Afghanistan zeigten. Er unterschied sich aber von seinem Vorgänger in der Frage, wie die amerikanische Militärmaschinerie eingesetzt werden sollte. Bush hatte das Militär nicht wie von der Powell Doctrine gefordert "[as] a last resort"108 verwendet, und den Schwerpunkt von „preemptive” auf "preventive” gelegt. Damit war für eine Kriegserklärung keine unmittelbare Bedrohung mehr notwendig gewesen, sondern es genügte bereits, wenn diese zu einem späteren Zeitpunkt unmittelbar („,imminent”) sein könnte. Gemäß dieses Kalküls sollten (unter Umständen noch gar nicht konkret bestehende) Gefahren frühest möglich, auf jeden Fall aber unter „favorable conditions", bekämpft werden. ${ }^{109}$ In diesem Konzept lässt sich die Berechtigung zum Krieg schon anhand überzeugend vorgetragener Vermutungen und somit ganz ohne handfeste und unwiderlegbare Beweise konstruieren. Bestes Beispiel war der IrakKrieg von 2003.

Präsident Obamas Vorgehen war anders, was im Falle Libyens deutlich wurde, wo es wenige Tage nach Mubaraks Rücktritt ebenfalls zu Tumulten kam. Libyens Staatschef Muammar al-Ghaddafi setzte seine Sicherheitskräfte umgehend und mit äußerster Brutalität gegen die Aufständischen ein. Der Konflikt eskalierte weiter und obwohl es zeitweise so schien, als könnten die Rebellen den Diktator selbst stürzen, wendete sich das Blatt wenig später wieder zu dessen Gunsten, womit die unmittelbare Gefahr im Raum stand, dass Ghaddafi seine Warnung, ",die Ratten' auszurotten"110, binnen kurzer Zeit wahrmachen könnte.

Obamas Berater waren sich über ein amerikanisches Eingreifen uneins. Verteidigungsminister Gates war zutiefst skeptisch „toward humanitarian intervention”111, wie sie etwa von der US-Botschafterin bei den Vereinten Nationen, Susan Rice, vertreten wurde ${ }^{112}$ und sah auch das nationale Interesse der USA nicht wesentlich berührt. Ein dritter

106 Mann, The Obamians, S. 260.

107 Ebd., S. 279.

108 LaFeber, The Rise and Fall, S. 73.

109 Szabo, Parting Ways, S. 65.

110 Lüders, Tage des Zorns, S. 92.

111 Mann, The Obamians, S. 288

112 Sanger, Confront and Conceal, S. 341 
Erklärungansatz für das letztliche amerikanische Militärengagement wäre die Stärkung der transatlantischen Bande. Frankreich, Deutschland und Großbritannien waren den USA in Afghanistan zur Seite gestanden. Nun baten Frankreich und Großbritannien die Vereinigten Staaten um Unterstützung. Ihnen diese zu verweigern, hätte wohl zu einem erneuten Abkühlen der Beziehungen geführt. Diese Episode ist ein weiteres Signal, dass der multilaterale Internationalismus wieder zum Leitmotiv der US-Außenpolitik wurde.

\section{Schluss}

Die in dieser Ausarbeitung untersuchten, nach dem Zweiten Weltkrieg entstandenen multilateralen Bündnisstrukturen waren mit dem Marshall-Plan und der EGKS wirtschaftlicher Natur und wiesen im Falle von NATO und EVG einen militärischen Charakter auf. Die USA beschränkten sich nach Möglichkeit auf die Festlegung der Rahmenbedingungen, mischten sich aber etwa dann ein, wenn Frankreich und Großbritannien Deutschland nicht wie im gewünschten Maße einzubinden bereit waren. Achesons Ungeduld über die langsamen Fortschritte der französischen EVG-Verhandlungen zeigten aber auch, dass sich die amerikanischen Europa-Vorstellungen den Ländern des Kontinents nicht aufoktroyieren ließen. Die USA benutzten zwar ihre wirtschaftliche und finanzielle Macht als Druckmittel, konnten diese aber nur mit Fingerspitzengefühl und im Rahmen von Verhandlungen ins Spiel bringen. Verhandlungen setzen Kompromissbereitschaft und mehr oder minder ebenbürtige Partner voraus. Der multilaterale Internationalismus setzte also abstrakt gesprochen auf kooperative Partnerschaftsverhältnisse, im konkreten Fall also die NATO oder die EGKS. Diese Bündnissysteme kombinierten Rechte und Pflichten der Mitglieder und teilten die Lasten, die mit dem Schutz Europas und dem Wiederaufbau des Kontinents einhergingen.

Mit George W. Bush und dem Einzug des (neo-) konservativen Denkens ins Weiße Haus wurden die Nachteile betont, die mit der Einbindung in internationale Bündnisstrukturen einhergingen. Die Vorteile schienen aus Bushs Perspektive den Nachteil, keine freie Hand in der Außenpolitik zu haben und Rücksichten auf Bündnispartner nehmen zu müssen, nicht aufzuwiegen. Bushs Kalkül, entschlossenes Voranschreiten allein würde die Verbündeten veranlassen, den USA in den Krieg zu folgen, war mit den europäischen partnerschaftlichen Vorstellungen nicht zu vereinbaren. Diese Einstellung des Präsidenten erklärt sich einerseits aus der enormen ökonomischen, politischen und militärischen Macht der Vereinigten Staaten zu Beginn des 21 . Jahrhunderts, wurde aber vermutlich auch durch die breite internationale Zustimmung bestärkt, die den USA im Golfkrieg von 1990/91 zugekommen war. Allerdings wäre das aber ein historisch unzulänglicher Vergleich, ging doch 2003 vom Irak, entgegen Regierungsangaben, keine unmittelbare Gefahr aus. Der irakische Überfall auf Kuwait 1990 ist denn eher mit 9/11 zu vergleichen, reagierten die USA doch beides Mal auf eine konkrete äußere Aggression. Von Trumans Multilateralismus war bei Bush Jr. jedenfalls wenig zu sehen.

Ob aber mit Obama eine Rückkehr zum multilateralen Internationalismus einherging, ist nicht pauschal zu beantworten, auch wenn der Militäreinsatz in Libyen diesen Schluss nahelegt. Ähnlich wie nach dem Zweiten Weltkrieg, legten die USA hier aber Wert auf 
eine Lastenteilung, indem sie nur anfänglich die Führung übernahmen, diese dann aber an Frankreich und Großbritannien übergaben, womit die NATO ihrem ursprünglichen Charakter als einer Institution partnerschaftlicher Kooperation gerecht wurde. Der hier von Obama vertretene internationale Führungsanspruch der USA setzte nicht wie bei Bush allein auf Entschlossenheit, sondern bedurfte des Konsenses der Bündnispartner. Die Truppenerhöhungen in Afghanistan zeigten aber prinzipiell auch, dass Obama eine als "richtig" erkannte Entscheidung ohne internationale Unterstützung umzusetzen bereit war. Trotz eines gewissen Pragmatismus vollzog sich mit Obama eine Akzentverschiebung in der US-Außenpolitik mit potenziell weitreichenden Folgen, wurde der Alleingang nunmehr doch zur ultima ratio, nachdem alle diplomatischen Stricke gerissen waren. Demgegenüber waren bei Bushs unilateralem Internationalismus die diplomatischen Bemühungen wenig mehr als eine Farce gewesen, um eine bereits gefällte Entscheidung zum Krieg in das Gewand der Legitimität und Rechtskonformität zu kleiden.

Diese Punkte legen den Schluss nahe, dass Bushs Außenpolitik tatsächlich nur eine temporäre Abweichung vom multilateralen Internationalismus Harry Trumans war. Dieser Eindruck wird noch durch die Verschiebung des amerikanischen Fokus von Europa auf Asien verstärkt, sind die USA doch angesichts des raschen wirtschaftlichen und militärischen Wachstums der Volksrepublik China auf Partner angewiesen. Zwar lässt sich hierüber noch kein historisches Urteil fällen, doch ließen sich die amerikanischeuropäischen Verhandlungen zur Schaffung einer gemeinsamen Freihandelszone durchaus als Anzeichen dafür deuten, dass die USA zumindest auf wirtschaftlichem Gebiet ein Gegengewicht zu China bilden wollen. Ein Gedanke, der zwar nicht zu beweisen, aber mit letzter Konsequenz zu Ende gedacht, die amerikanische Europapolitik als Teil einer Eindämmungspolitik gegenüber der Supermacht China erscheinen lässt. Diese Spekulationen beiseite lassend, lässt sich trotz allem feststellen, dass die USA aktuell ähnlich vorgehen wie nach 1945, als "Washington actively cultivated friends and allies because in a world with a superpower adversary it was dangerous to be without them"113.

Obamas Worte, als Präsidentschaftskandidat bei seinem Berlinbesuch 2008 geäußert, sind mehr als nur positive Bestätigung der These, dass Bushs Außenpolitik lediglich eine temporäre Abweichung vom multilateralen Internationalismus war. Sie geben die Richtung der US-Außenpolitik für das 21. Jahrhundert vor, wie sie auch die Marschrichtung nach dem Zweiten Weltkrieg beschreiben: „Partnership and cooperation among nations [...] is [...] the only way"114.

\section{Literatur}

Acheson, Dean, Present at the creation. My years in the State Department, New York 1987.

Blair, Tony, A Journey, London 2011.

113 Daalder/Lindsay, America unbound, S. 10

114 The New York Times, Obama's Speech in Berlin, 24.07.2008, [http://www.nytimes.com/2008/07/24/us/ politics/24text-obama.html?pagewanted=all\&_r=0], eingesehen 07.10.2013. 
Brzezinski, Zbigniew/Scowcroft, Brent, America and the World. Conversations on the Future of American Foreign Policy, New York 2008.

Bundeswehr, Chronologie des Einsatzes in Afghanistan ISAF, 04.10.2013, [http:// www.einsatz.bundeswehr.de/portal/a/einsatzbw/lut/p/c4/LcgxDoAgDEbhs3gBurt5 C3Uxv 1 qhgRQjVRJOr4N50_dopi_FIx4mWZFopGmTfq l urTsvLFpg7SOi3ZzSV9gaOxwQKUY1G3hypoXKTjojEP3AjH-WW0!/], eingesehen 07.10.2013.

CNN, Obama speech: ,Yes, we can change', 27.01.2008, [http://edition.cnn.com/2008 /POLITICS/01/26/obama.transcript/], eingesehen 06.10.2013.

C-Span Video Library, U.S. President Speech, 12.09.2002, [http://www.c-spanvideo.org/ program/172611-2], eingesehen 20.09.2013.

Daalder, H./Lindsay, James M., America unbound. The Bush revolution in foreign policy, New Jersey 2005.

Daalder, H./Stavridis, James G., NATO's Victory in Libya. The Right Way to Run an Intervention, O. D., [http://www.foreignaffairs.com/articles/137073/ -h-daalder-andjames-g-stavridis/natos-victory-in-libya], eingesehen 06.10.2013.

DeYoung, Karen, Soldier. The Life of Colin Powell, New York 2006.

Fursdon, Edward, The Role of the European Defense Community in European Integration, in: Heller, Francis H./Gillingham, John R. (Hrsg.), NATO: The Founding of the Atlantic Alliance and the Integration of Europe, Basingstoke-London 1992, S. 213-240.

G20, What is the G20, o. D., [http://www.g20.org/docs/about/about_G20.html], eingesehen 02.10.2013.

Gaddis, John Lewis, Der Kalte Krieg. Eine Neue Geschichte, München 2007.

Gersdorff, Gero von, Die Gründung der Nordatlantischen Allianz (Entstehung und Probleme des Atlantischen Bündnisses), München 2009.

Gordon, Michael R./Sanger, David E., A Nation Challenged: Iraq; Powell Says U.S. Is Weighing Ways To Topple Saddam Hussein, 13.02.2002, [http://www.nytimes.com/20 02/02/13/world/a-nation-challenged-iraq-powell-says-us-is-weighing-ways-to-topplehussein.html?pagewanted=2], eingesehen 17.09.2013.

Guthrie-Shimizu, Sayuri, Japan, the United States, and the Cold War, 1945-1960, in: Leffler, Melvyn P.Nestad, Odd Arne (Hrsg.), The Cambridge History of the Cold War I, Cambridge 2010, S. 244-265.

G20, What is the G20, o. D., [http://www.g20.org/docs/about/about_G20.html], eingesehen 02.10.2013.

Hitchcock, William I., The Marshall Plan and the creation of the West, in: Leffler, Melvyn P./ Westad, Odd Arne (Hrsg.), The Cambridge History of the Cold War I, Cambridge 2010 , S. 154-174. 
Hogan, Michael J., Europäische Integration und deutsche Reintegration: Die Marshallplaner und die Suche nach Wiederaufbau und Sicherheit in Europa, in: Maier, Charles S./Bischof, Günter (Hrsg.), Deutschland und der Marshall-Plan, Baden-Baden 1992, S. 139-199.

Hogrefe, Jürgen, Gerhard Schröder. Ein Porträt, Berlin 2002.

Indyk, Martin S./Lieberthal, Kenneth G./O’Hanlon, Michael E., Bending History. Barack Obama's Foreign Policy, Washington D.C. 2012.

Information Clearing House, Defense Strategy for the 1990s: The Regional Defense Strategy, Januar 1993, [http://www.informationclearinghouse.info/pdf/naarpr_Defense. pdf], eingesehen 10.09.2013.

International Security Assistance Force, Troop Numbers and Contributions, 01.08.2013, [http://www.isaf.nato.int/images/stories/File/Placemats/2013-08-01\%20ISAF\%20Place mat-final.pdf], eingesehen 14.09.2013.

Isaacson, Walter/Thomas, Evan, The Wise Men. Six Friends and the World they Made. Acheson, Bohlen, Harriman, Kennan, Lovett, McCloy, New York 1986.

Johnstone, Andrew, Isolationism and internationalism in american foreign policy, in: Journal of Transatlantic Studies 9 (201 1), Nr. 1, S. 7-20, hier S. 14, [http://www.tandfo nline.com/ doi/pdf/10.1080/14794012.2011.550772\#.VRLjK-Edldw], eingesehen 06.10.2013.

Kennan, George F., 861 1.00/2 - 2246: Telegram. The Charge in the Soviet Union (Kennan) to the Secretary of State, o.D., [http://www2.gwu.edu/ nsarchiv/coldwar/doc uments/ episode-1/kennan.htm], eingesehen 11.05.2013.

Kleber, Claus, Amerikas Kreuzzüge. Was die Weltmacht treibt, München 2005.

LaFeber, Walter, The Rise and Fall of Colin Powell and the Powell Doctrine, in: Political Science Quarterly 124 (2009), Nr. 1, S. 71-93, hier S. 73, [http://onlinelibrary. wiley.com/ doi/10.1002/j.1538-165X.2009.tb00642.x/abstract], eingesehen 29.08.2013.

Lambakis, James Steven, Winston Churchill. Architect of Peace. A Study of Statesmanship and the Cold War (Contributions in Political Science), Westport 1993.

Large, David Clay, Grand Illusions: The United States, the Federal Republic of Germany, and the European Defense Community, 1950-1954, in: Diefendorf, Jeffry M./Frohn, Axel/Rupieder, Hermann-Josef (Hrsg.), American Policy and the Reconstruction of West Germany, 1945-1955, (Publications of the German Historical Institute), Cambridge 1993 , S. 375-394.

Larres, Klaus, Eisenhower, Dulles und Adenauer. Bündnis des Vertrauens oder Allianz des Mißtrauens?. (1953-1961), in: Larres, Klaus/Oppelland, Torsten (Hrsg.), Deutschland und die USA im 20. Jahrhundert. Geschichte der politischen Beziehungen, Darmstadt 1997, S. 119-151. 
Leffler, Melvyn P., The emergence of an American grand strategy, 1945-1952, in: Leffler, Melvyn P./Westad, Odd Arne (Hrsg.), The Cambridge History of the Cold War I, Cambridge 2010.

Loth, Wilfried, Der Weg nach Europa. Geschichte der europäischen Integration 19391957, Göttingen $1996^{3}$.

Lüders, Michael, Tage des Zorns. Die Arabische Revolution verändert die Welt, München 2011.

Mai, Gunther, American Policy toward Germany and the Integration of Europe, 19451955, in: Diefendorf, Jeffry M./Frohn, Axel/Rupieder, Hermann-Josef (Hrsg.), American Policy and the Reconstruction of West Germany, 1945-1955, (Publications of the German Historical Institute), Cambridge 1993, S. 85-109.

Maier, Charles S., Es geht um die Zukunft Deutschlands und damit um die Zukunft Europas, in: Maier, Charles S./Bischof, Günter (Hrsg.), Deutschland und der Marshall-Plan, Baden-Baden 1992, S. 13-52.

Mann, James, Rise of the Vulcans. The History of Bush's War Cabinet, New York 2004.

Ders., The Obamians. The Struggle Inside the White House to Redefine American Power, New York 2012.

National Security Strategy Archive, The National Security Strategy of the United States of America, S. 25, [http://nssarchive.us/NSSR/2002.pdf], eingesehen 09.09.2013.

Nordhausen, Frank/Schmid, Thomas (Hrsg.), Die Arabische Revolution, Berlin 2011.

Overhaus, Marco, Die deutsche NATO-Politik. Vom Ende des Kalten Krieges bis zum Kampf gegen den Terrorismus (Außenpolitik und Internationale Ordnung), Baden-Baden 2009, S. 262.

Perkins, Dexter, Fundamental Principles of American Foreign Policy, in: Annals of the American Academy of Political and Social Sciences 218 (1941), Heft 6, S. 9-19, [http:// ann.sagepub.com/content/218/1/9. extract], eingesehen 26.04.2013.

Rienäcker, Cai, Freier Handel zwischen zwei Kontinenten, 13.02.2013, [http://www.tag esschau.de/wirtschaft/freihandelszone102.html], eingesehen 09.10.2013.

Rudolf, Peter, Das »neue» Amerika. Außenpolitik unter Barack Obama, Berlin 2010.

Sanger, David E., Confront and Conceal. Obama's Secret Wars and Surprising Use of American Power, New York 2012.

Schöttli, Thomas U., USA und EVG. Truman, Eisenhower und die Europa-Armee (Europäische Hochschulschriften), Bern 1994.

Schwabe, Klaus, Bündnispolitik und Integration 1949-1956, in: Maier, Klaus A./ Wiggershaus, Norbert (Hrsg.), Das Nordatlantische Bündnis 1949-1956, (Beiträge zur Militärgeschichte), München 1993, S. 71-87. 
Schwartz, Thomas A., Europäische Integration und special Relationship - Zur Durchführung des Marshall-Planes in der Bundesrepublik Deutschland, 1948- 1951, in: Maier, Charles S./Bischof, Günter (Hrsg)., Deutschland und der Marshall-Plan, BadenBaden 1992, S. 201-249.

Schwarz, Klaus-Dieter, Die NATO ist tot - es lebe die NATO, in: Die Welt, 14.5.2002, zit. n. Overhaus (2009).

Szabo, Stephen F., Parting ways. The crisis in German-American relations, Washington D.C. 2004.

The New York Times, Excerpts From Pentagon's Plan: ,Prevent the Re-Emergence of a New Rival', 08.03.1992, [http://www.nytimes.com/1992/03/08/world/excerpts-frompentagon-s-plan-prevent-the-re-emergence-of-a-new-rival. html?pagewanted=all\&src=p $\mathrm{m}$, eingesehen 10.09.2013.

The New York Times, Obama's Speech at Camp Lejeune, N.C., 27.02.2009, [http:// www.nytimes.com/2009/02/27/us/politics/27obama-text. html?pagewanted=1 \&_r=0], eingesehen 06.10.2013.

The New York Times, Obama's Speech in Berlin, 24.07.2008, [http://www.nytimes. com/2008/07/24/us/politics/24text-obama.html?pagewanted=all\&_r=0], eingesehen 07.10. 2013.

The White House, Remarks by the President in Address to the Nation on the Way Forward in Afghanistan and Pakistan, 01.12.2009, [http://www.whitehouse.gov/thepress-office/remarks-president-address-nation-way-forward-afghanistan-and-pakistan], eingesehen 07.10.2013.

Trubowitz, Peter, Regional Shifts and US foreign policy, in: Cox, Michael/Stokes, Doug (Hrsg.), US Foreign Policy, New York 2008, S. 145-162.

United Nations Security Council, Resolution 1368 (2001), 12.09.2001, [http://www. un. org/en/ga/search/view_doc.asp?symbol=S/RES/1368\%282001\%29], eingesehen 13.09.2013.

United Nations, The Status of Nuclear Inspections in Iraq. Statement to the United Nations Security Council, 27.01.2003, [http://www.un.org/news/dh/iraq/elbaradei27jan 03.htm], eingesehen 20.09.2013.

United States Senate, U.S. Senate Roll Call Votes $110^{\text {th }}$ congress - $1^{\text {st }}$ session. A bill to express the sense of Congress on Iraq, 17.02.2007, [http://www.senate.gov/legislate ve/LIS/roll_call_lists/roll_call_vote_cfm.cfm?congress $=110 \&$ session= 1 \&vote=00051 ], eingesehen 23.09.2013.

U.S. Government Printing Office, Compilation of Presidential Documents, Addresses and Remarks, Address Before a Joint Session of the Congress on the State of the Union, 29.01.2002, S. 135, [http://www.gpo.gov/fdsys/pkg/WCPD-2002-02-04/pdf/WCPD2002-02-04-Pg133-3.pdf], eingesehen 13.09.2013. 
U.S. Government Printing Office, Public and Private Laws, Authorization for Use of Military Force Against Iraq Resolution of 2002, 16.10.2002, [http://www.gpo.gov/fdsy s/pkg/ PLAW-107publ243/pdf/PLAW-107publ243.pdf], eingesehen 20.09.2013.

Volkmann, Hans-Erich, Adenauer, Frankreich und die Europäische Verteidigungsgemeinschaft, in: Wilkens, Andreas (Hrsg.), Interessen verbinden. Jean Monnet und die europäische Integration der Bundesrepublik Deutschland, (Pariser Historische Studien), Bonn 1999, S. 161-186.

Wall, Irwin M., France and the North Atlantic Alliance, in: Heller, Francis H./Gillingham, John R. (Hrsg.), NATO: The Founding of the Atlantic Alliance and the Integration of Europe, Basingstoke-London 1992, S. 45-56.

Woodward, Bob, Bush at War. Amerika im Krieg, Stuttgart-München 2003.

X, The Sources of Soviet Conduct, in: Foreign Affairs 25 (1947), Nr. 4, S. 566-582, hier S. 575, [http://www.jstor.org/discover/10.2307/20030065?sid=21106240177913\&u id=3 8722 \&uid=591021 6\&uid=67\&uid=62\&uid=38721 \&uid=3\&uid=70\&uid=2\&uid=2134\&u $i d=3737864 \&$ uid=5910616], eingesehen 12.05.2013.

Thomas Peter Salzmann ist Absolvent des BA Geschichte der Universität Innsbruck und Student des MA Geschichtswissenschaften im 2. Semester an der Universität Tübingen. thomas-peter.salzmann@student.uni-tuebingen.de

\section{Zitation dieses Beitrages}

Thomas Peter Salzmann, Die Außenpolitik der Vereinigten Staten von Amerika und inr Bekenntnis zum multilateralen Internationalismus, in: historia.scribere 7 (2015), S. 169196, [http://historia.scribere.at], 2014-2015, eingesehen 1.3.2015 (=aktuelles Datum).

(C) Creative Commons Licences 3.0 Österreich unter Wahrung der Urheberrechte der Autorlnnen. 